\title{
The Role of AEBP1 in Sex-Specific Diet-Induced Obesity
}

\author{
Lei Zhang, Shannon P Reidy, Tara E Nicholson, Hyun-Jeong Lee, Amin Majdalawieh, \\ Chris Webber, Bruce R Stewart, Peter Dolphin, ${ }^{\dagger}$ and Hyo-Sung Ro
}

\begin{abstract}
Obesity is an important risk factor for heart disease, diabetes, and certain cancers, but the molecular basis for obesity is poorly understood. The transcriptional repressor AEBPI, which functions as a negative regulator of PTEN through a protein-protein interaction, is highly expressed in the stromal compartment of adipose tissues, including proliferative preadipocytes, and its expression is abolished in terminally differentiated, nonproliferative adipocytes. Here we show that transgenic overexpression of AEBP1 during adipogenesis coupled with a high-fat diet (HFD) resulted in massive obesity in female transgenic (AEBP ${ }^{T G}$ ) mice via adipocyte hyperplasia. AEBP1 levels dynamically changed with aging, and HFD induced AEBP1 expression in females. Thus, HFD-fed AEBPITG females display hyperinduction of AEBPI and a marked reduction of PTEN level with concomitant hyperactivation of the survival signal in white adipose tissue. Our results suggest that AEBP1 plays a key functional role in in vivo modulation of adiposity via fatcell proliferation and is involved in a sex-specific susceptibility to diet-induced obesity by the estrogen signaling pathway.
\end{abstract}

Online address: http://www.molmed.org

doi: 10.2119/2005-00021.Ro

\section{INTRODUCTION}

Obesity results from an imbalance between energy intake and energy expenditure that leads to excess storage of calories as triglyceride. Recent progress in understanding how energy balance and body weight are regulated has been marked by the discovery of hormones and the neural pathways operating downstream of these hormones that bring about physiological changes (1). A complementary approach for the treatment of obesity focuses on the periphery, the actual tissue where the obese phenotype arises $(2,3)$.

Adipogenesis is characterized by an increased lipogenic capacity, a change from a fibroblast morphology to the unilocular appearance of mature adipocytes, and an alteration in the expression levels of hundreds of proteins. We have previously focused on the regulation of $a P 2$ (encodes adipocyte lipid-binding protein) gene expression (4) and defined a cis controlling element (AE-1) and trans-acting factors, including $\mathrm{C} / \mathrm{EBP} \alpha$ (5), human preadipocyte factors (6), and AEBP1 (7), which are involved in either positive or negative regulation of $a P 2$ expression.

AEBP1 is a transcriptional factor with carboxypeptidase (CP) activity that is vital for transcriptional repression function (7). The binding of DNA is a novel function for this type of regulatory $\mathrm{CP}(8)$. Yeast two-hybrid studies have revealed that the $\gamma 5$ subunit of a heterotrimeric $G$ protein is an interacting partner of AEBP1. G $\gamma 5$ can prevent AEBP1 from binding DNA and inhibit transcriptional repression activity of AEBP1, and this derepression activity of G 55 is modulated by adipogenic stimulation (9). This is the first report of a signal-transducing molecule being involved, by direct protein-protein interaction, with the regulation of transcription during adipogenesis.
AEBP1 interacts with another important signal-transducing molecule, mitogen-activated protein kinase (MAPK). We provided new information on the signaling pathway that links AEBP1 and MAPK in adipogenesis (10). Overexpression of AEBP1 increases and sustains MAPK (ERK1/2) activation in 3T3-L1 preadipocytes. The modulation of ERK1/2 activation, which is controlled by the protective effect of AEBP1 toward the MAPK-specific phosphatase MKP-3, is a critical part of the molecular mechanism behind adipogenesis. Recently, MAPK has been shown to regulate the transcriptional activity of AEBP1 by a novel dual mechanism, in which MAPK interaction enhances and subsequent phosphorylation decreases the DNA-binding ability of AEBP1 (11). Therefore, a complex regulatory loop exists between AEBP1 and MAPK.

The AEBP1 gene extends over $10 \mathrm{~kb}$, has 21 exons, and gives rise to 2 mRNAs (AEBP1 and ACLP) by alternative splicing, in which the 9th intron is retained in the mature AEBP1 transcript. Thus, ACLP encodes an additional 380 amino acids $\mathrm{NH}_{2}$-terminal to the first ATG codon of AEBP1, which is located in exon 10 (12). ACLP contains a signal peptide within the $\mathrm{NH}_{2}$-terminal 380 amino acids, and its expression up-regulates during vascular smooth muscle cell differentiation (13). AEBP1 expression persists during 3T3-L1 preadipocyte differentiation, and then it ends only at the terminal stage of adipocyte differentiation $(7,10)$. In vivo, AEBP1 is abundantly expressed in white adipose tissue (WAT), most likely from nonadipocyte cells, including preadipocytes resident within adipose tissue (stromal-vascular fraction), but its expression is abolished in the mature fat cells purified from WAT (12). Therefore, AEBP1 expression persists during adipogenesis and is abolished only in terminally differentiated adipocytes.

Department of Biochemistry \& Molecular Biology, Faculty of Medicine, Dalhousie University, Halifax, NS, B3H 1X5 Canada 
AEBP1 was identified as an interacting partner of the tumor suppressor PTEN (14). PTEN plays a key role in mammalian growth control $(15,16)$. It antagonizes the activity of growth factorstimulated phosphatidylinositol 3-kinase (PI3K) that controls many downstream cellular processes including cell growth, apoptosis, and cell motility. By its specific lipid-phosphatase activity responsible for removal of the phosphate in the D3-phosphate group of the second messenger phosphatidylinositol 3,4,5trisphosphate $\left(\mathrm{PIP}_{3}\right)$, PTEN down-regulates the prosurvival serinethreonine kinase Akt (PKB). We have recently demonstrated that AEBP1 and PTEN interact in mammalian cells, and this interaction promotes PTEN degradation. Thus, PTEN activity is negatively correlated with the amount of AEBP1 protein. Furthermore, our results with AEBP1-null mice suggest that the negative regulation of PTEN by AEBP1, in conjunction with the control on proliferative potential of preadipocytes, is critical to modulation of in vivo adiposity through its influence on adipocyte survival. Homozygous AEBP1-deficient mice displayed reduced adipose tissue mass, with enhanced apoptosis and suppressed survival signal coinciding with diminished proliferative expansion of preadipocytes exhibiting lowered basal MAPK activity (submitted).

Here we present further evidence for the regulatory role of AEBP1 in the control of in vivo modulation of adiposity through its negative regulation of PTEN function. We investigated the physiological role of the PTEN control by transgenic overexpression of AEBP1 during adipogenesis. Our results suggest that AEBP1 has 2 distinct estrogen-mediated regulatory roles in modulation of adiposity via fat cell number and size, and that AEBP1 is a critical regulator of energy metabolism.

\section{MATERIALS AND METHODS}

\section{Cell Culture, Plasmids, Transfection, and Reporter Assays}

3T3-L1 and antisense preadipocytes were cultured as described previously (10). CHO and HC11 cells were cultured in 90-mm dishes and transfected at $70 \%$ to $80 \%$ confluence using Polyfect (Qiagen) or at $90 \%$ to $95 \%$ confluence using Lipofectamine 2000 (Invitrogen). Chimeric plasmids carrying selected promoter regions of the AEBP1 gene fused to a firefly luciferase gene (LUC) were constructed by inserting DNA regions extending different distances $5^{\prime}$ from the AEBP1 promoter into the promoterless reporter vector pGL2-Basic (Promega). Luciferase reporter activity was assessed using a luciferase assay system (Promega Corp., Madison, WI, USA) according to the manufacturer's instructions. In brief, transiently transfected $\mathrm{CHO}$ cells in 12-well plates were washed in cold PBS and subsequently lysed in passive lysis buffer. Thirty microliters of total cell extract was used to measure reporter activity using the BMG FLUOstar Galaxy microplate reader (BMG Labtechnologies, Durham, NC, USA). $\beta$-Galactosidase assay was performed as previously described (7).

\section{Transgenic Animals}

The fat-specific AEBP1 transgene was constructed using the 5.4-kb DNA fragment containing the fat-specific promoter/enhancer (17) from the fatty acid-binding protein gene $a P 2$, and it was ligated to a 2.6-kb mouse AEBP1 cDNA. The aP2 promoter/ enhancer directs expression in adipocytes as well as macrophages (18). The transgene plasmid was digested with Hind III and NdeI to release the 8.7-kbp aP2-AEBP1 transgene for the injection. The construct was injected into the pronucleus of fertilized zygotes from FVB mice and transferred to pseudopregnant females. A total of 35 pups were born from 4 mice impregnated with blastocysts that had been injected with the AEBP1 transgene construct. Eight pups acquired the transgene, as shown by PCR analysis of tail DNA. These 8 founders were mated, and all the mating pairs produced first sets of $F_{1}$ mice. Pups were weaned at the age of 3 weeks. Mice positive for the transgene were determined by tail DNA PCR using the sense primer in exon 18 (5'-GGACTACACCAGCGGCATGG-3') and the antisense primer from exon 21 (5'-GCGTGAGCTGTCACACGGTA-3'). This primer pair amplifies a 360-bp fragment of cDNA and a genomic sequence of $\sim 900 \mathrm{bp}$. One transgenic littermate from each founder was killed at the age of 7 weeks to examine tissue-specific expression of the AEBP1transgene. Ten different tissues were isolated, and total RNA was prepared from these tissues.

\section{Animal Experiments}

All animal experiments were performed according to procedures approved by the institutional Animal Care Committee. For ovariectomy, a small midline dorsal skin incision was made approximately halfway between the middle of the back and base of the tail, half to two-thirds of the way down the side of the body. The ovary, surrounded by a variable amount of fat, was located and pulled out through the muscle incision by grasping the periovarian fat. With pointed scissors, the junction between the Fallopian tube and the uterine horn, together with all the accompanying blood vessels and fat, was severed with a single cut, and the horn was returned to the abdominal cavity. When there was considerable fat obscuring the ovary, the connection between the Fallopian tube and the uterine horn was cut first, followed by a cut through the ovarian blood vessels and accompanying fat. The ovaries were not touched in mice having a sham operation.

Three-week-old AEBP1-transgenic and nontransgenic control mice were weaned on a standard rodent diet or a high-fat diet (45\% total calories in the form of fat; D12451; Research Diets, New Brunswick, NJ, USA). Mice had access to food and water ad libitum and were weighed every week up to 32 weeks. The amount of food consumed was determined by weighing the food from each cage weekly and normalizing to the number of mice in each cage. The values are presented as the average amount of food consumed per mouse.

Glucose levels were measured in all animals every 3 weeks. Blood samples were obtained by snipping the tail with surgical scissors and using a Bayer Elite XL glucometer (Toronto, ON, Canada) to determine glucose levels in the blood droplet. Fed and fasted glucose levels were determined at the same time of day throughout the study; for the fasted values, the animals were first deprived of food for $12 \mathrm{~h}$. Glucose values were averaged for each group and reported as millimoles per liter.

Following completion of the 32-week high-fat diet studies, the mice were deeply anesthetized by injection of sodium pento- 
barbital (Somnotol) and blood was obtained by intracardiac puncture. Blood samples were allowed to clot overnight at $4^{\circ} \mathrm{C}$ then spun down at $2000 \mathrm{~g}$ for $20 \mathrm{~min}$ to obtain the serum fraction. Insulin and leptin levels were measured using ELISA kits (Crystal Chemical, Chicago, IL, USA) as outlined in the manufacturer's instructions; levels are reported as nanograms per milliliter for insulin and nanograms per gram body weight for leptin. Whole serum samples obtained from mice were also analyzed for total lipids as described previously (19). Serum lipids were analyzed on a Hewlett Packard 5890 Gas Chromatograph using a siliconized glass column packed with 3\% SP-2100 on 100/20 Supelcopart. Samples were eluted using UHP helium as the carrier gas with a flow rate of $33 \mathrm{~mL} / \mathrm{min}$. Tridecanoin (Sigma, Oakville, ON, Canada) was used as the internal control for these studies. Samples were detected using flame ionization detection, and total serum cholesterol and triacylglycerides are reported as milligrams per deciliter.

\section{Insulin and Glucose Tolerance Tests}

All mice were fasted for $16 \mathrm{~h}$. Normal fasted and fed blood glucose levels were measured using LifeScan Sure-Step Glucometer. Each mouse received an injection of insulin (0.75 units $/ \mathrm{kg}$; Sigma). Blood samples were collected at 15, 30, 45, and $60 \mathrm{~min}$ postinjection. After $2 \mathrm{~d}$, the mice were fasted again for $16 \mathrm{~h}$. Each mouse received an injection of glucose ( $1 \mathrm{mg} / \mathrm{g}$; Invitrogen), and blood samples were collected as described above.

\section{In Vivo Insulin Stimulation}

All mice were fasted for $16 \mathrm{~h}$. Each mouse received an intraperitoneal injection of 40 to $50 \mathrm{mg} / \mathrm{kg}$ Somnotol (CDMV, St Hyacinthe, QC, Canada). When mice were heavily sedated, the surgical area was cleaned with sterile saline, and a lateral incision was made to open the abdomen. A surgical blade was used to separate dermis from intraperitoneal cavity. Mammary gland and WAT were removed as reference points before stimulation; dissection sites were cleaned with sterile saline. Insulin (5 units; Sigma) was injected directly to the inferior vena cava. The injection site was cleaned, and the surgery area was soaked with saline and covered in gauze during the 15-min stimulation period. Tissues were dissected at $15 \mathrm{~min}$ (previously determined as the optimal stimulation time point), and protein lysates were isolated and used for protein analysis by immunoblotting. Antibodies against AKT, p-AKT, and p-GSK-3ß (New England Biolabs, Ipswich, MA, USA) were used to determine activation of PI3K pathway by insulin stimulation.

\section{Fat Pad Weight, Cellularity, and Histology}

Mice were killed by cervical dislocation, and the visceral fat pads were dissected, weighed, and flash-frozen in liquid nitrogen for the cellularity experiments. Fat cell size and fat cell number were determined by the method of Hirsch and Gallian (20). WAT was dissected from 32-week-old mice on high-fat diets. Fat cell size was measured by counting the total number of osmium-fixed cells obtained from a known weight of tissue lipid, which was expressed in micrograms of lipid per cell. It was calculated by dividing the lipid weight of the fixed tissue sample by the total number of cells in the sample. Tissues were fixed in formaldehyde, embedded in paraffin, transversely sectioned (5- $\mu \mathrm{m}$ intervals), and stained with hematoxylin and eosin for histological analysis.

\section{RNA and Protein Analysis}

Total RNA from different mouse tissues was prepared with the RNA STAT-60 Solution (Tel-Test B; Tel-Test, Friendswood, TX, USA) according to the manufacturer's protocol. Twenty micrograms of total RNA from each tissue was loaded on a formaldehyde denaturating $1 \%$ agarose gel and blotted onto nylon transfer membrane (Micron Separations Inc.) as described (21). The filter was hybridized in QuikHyb solution (Stratagene, La Jolla, CA, USA) with ${ }^{32} \mathrm{P}$-labeled AEBP1 cDNA probe in a hybridization oven (Hybaid) for $3 \mathrm{~h}$ at $65{ }^{\circ} \mathrm{C}$. The filter was washed twice with $2 \times \mathrm{SSC} / 0.1 \%$ SDS for $15 \mathrm{~min}$ at room temperature and twice with $0.2 \times \mathrm{SSC} / 0.1 \% \mathrm{SDS}$ for $30 \mathrm{~min}$ at $65^{\circ} \mathrm{C}$ before exposure to $\mathrm{x}$-ray film overnight at $-70{ }^{\circ} \mathrm{C}$. Two micrograms of total RNA was used to synthesize cDNA using the Omniscript RT kit (Qiagen, Valencia, CA, USA). One-tenth of the RT reaction was used in the PCR reaction (HotStar Taq kit; Qiagen). Primer pair sets for leptin were forward primer, 5'-CCAAAACCCTCATCAAGACC-3', and reverse primer, 5'-CTCAAAGCCACCACCTCTGT-3'. The amplification cycle profile used was $95^{\circ} \mathrm{C}$ for $15 \mathrm{~min}$ followed by 35 cycles of $94{ }^{\circ} \mathrm{C}$ for $15 \mathrm{~s}, 60{ }^{\circ} \mathrm{C}$ for $1 \mathrm{~min}$, and $72{ }^{\circ} \mathrm{C}$ for $1 \mathrm{~min}$. Final 10 -min incubation at $72{ }^{\circ} \mathrm{C}$ finished off the profile. Products were separated out on a $1.4 \%$ agarose gel and visualized by UV light. The program Molecular Analyst (Bio-Rad, Hercules, CA, USA) was used for band density measurement.

Mouse tissue extracts were prepared by homogenizing in high-salt buffer (500 mM NaCl, $10 \mathrm{mM}$ Tris [pH 7.4], 1\% Triton X-100, 2 mM EDTA, 1 mM DTT, 1 mM PMSF, 1 mM Na$V_{3} \mathrm{VO}_{4^{\prime}}$ $1 \mathrm{mM}$ sodium molybdate, and protease inhibitors cocktail). Liver extracts were prepared by homogenizing in PBS ( $\mathrm{pH} 7.4$ ) containing $1 \%$ NP-40, $0.5 \%$ sodium deoxycholate, $0.1 \%$ SDS, and protease inhibitors cocktail. The precipitated samples or total protein extracts were separated on SDS-PAGE and transferred to nitrocellulose membranes. The membranes were incubated with blocking buffer for $1 \mathrm{~h}$ at room temperature, and then incubated with specific primary antibodies for $1 \mathrm{~h}$ at room temperature or overnight at $4^{\circ} \mathrm{C}$. Membranes were incubated with HRP-conjugated secondary antibody (1:3000) for $1 \mathrm{~h}$ at room temperature and analyzed by ECL blotting system (Amersham).

\section{RESULTS AND DISCUSSION}

\section{AEBP1 Modulates In Vivo Adiposity}

Adipocyte number may be modulated by apoptosis of preadipocytes and adipocytes (22-25), which is controlled by the PTEN-PI3K signaling pathway. AEBP1 promotes PTEN degradation, and AEBP1-null mice display reduced adipose tissue mass with enhanced apoptosis and suppressed survival signal (submitted). To further substantiate AEBP1's role as a key modulator of in vivo adiposity, we generated transgenic mice in which AEBP1, but not ACLP, expression was artificially maintained at a high level during adipogenesis. In vivo, AEBP1 is abundantly 
A
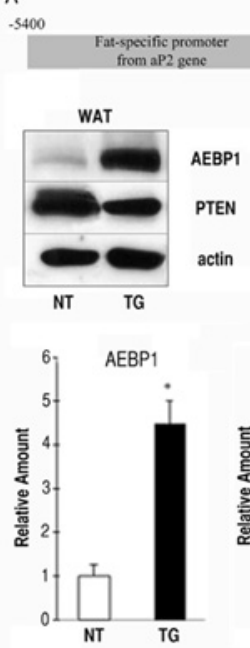

B
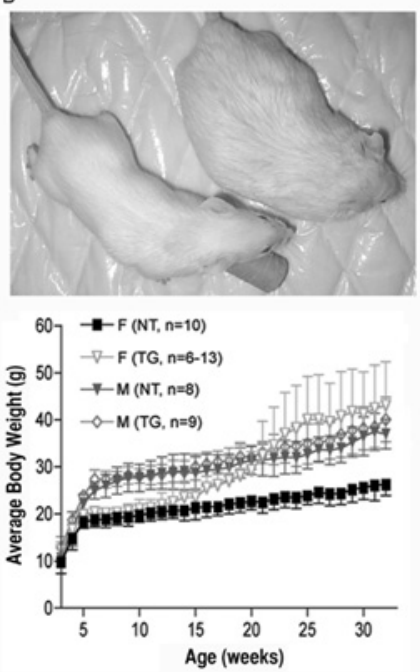

C

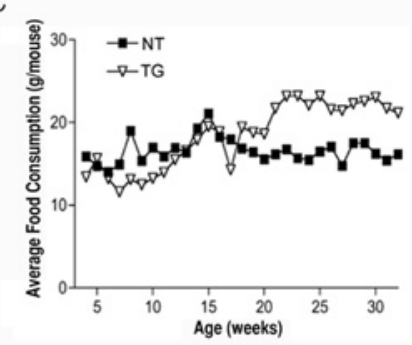

D

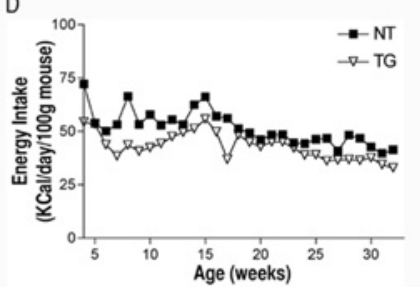

E

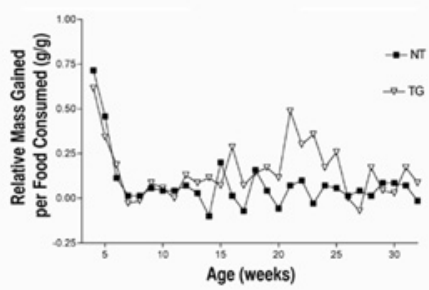

$\mathrm{F}$

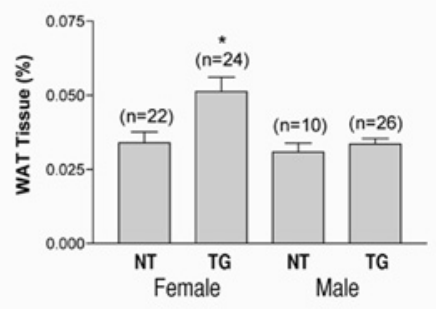

Figure 1. Transgenic overexpression of AEBPI in adipose tissue results in decreased expression of PTEN and increased adiposity in females on HFD. (A) Schematic representation of the fat-specific AEBP1 transgene. Expression of AEBPI and PTEN were examined by immunoblotting with anti-AEBPl or anti-PTEN antibody in the transgenic (TG) and nontransgenic (NT) littermates (32 weeks). Liver samples are shown as a control. Actin was used as a loading control. The average values of AEBPI and PTEN expression in WAT from TG $(n=7)$ and NT $(n=6)$ mice are represented as the mean \pm SD. ${ }^{*} P<0.0001$. (B) Growth patterns of TG and NT mice on HFD. Each data point represents the mean \pm SD of at least 6 mice. (C) Total food consumption per group in a cage was measured. Each data point represents the mean value per mouse. (D) Total energy intake per group in a cage was calculated. Each data point represents the mean value per 100 grams body weight. (E) Average feed efficiency was calculated with the mean value of weight gain in panel $B$ and the mean value of food consumption in panel C. (F) The average values of total WAT (gonadal and visceral) in TG and NT mice fed on $H F D$. Each bar represents the mean \pm SEM of the percent body weights. $* P<0.01$ compared with female NT mice.

expressed in the stromal compartment of adipose tissues, including proliferative preadipocytes, but it is abolished in mature fat cells (12). We anticipated that constitutive overexpression of AEBP1 during adipogenesis would maintain lower levels of PTEN that would promote the survival effect on differentiating fat cells,

which would eventually lead to obesity associated with adipocyte hyperplasia. The fat-specific AEBP1 transgene (Figure 1A, top panel) was constructed using a 5.4-kb DNA fragment containing the fat-specific promoter/enhancer from the fatty acid-binding protein gene $a P 2$, whose expression is highly induced during adipocyte differentiation (4-6). Although the 5.4-kb DNA fragment contains the AEBP1-binding sequence AE-1 at the proximal promoter region (6), which would have a potential negative feedback inhibition effect, 2 transgenic lines overexpressing AEBP1 in the adipose tissues were identified. The amount of AEBP1 protein in WAT was about 4.5-fold higher in one of the transgenic (TG) mouse lines than in a nontransgenic (NT) littermate control (Figure 1A). Initial construction of the AEBP1-transgene without the proximal promoter region, including the AE-1 sequence, in the 5.4-kb DNA fragment did not produce any transgenic mice with tissue-specific overexpression of AEBP1. We examined the impact of AEBP1 overexpression on PTEN protein levels and observed a significant decrease in the PTEN protein level in the WAT of TG mice. No such changes were observed in liver (Figure 1A).

We assessed the effect of targeted overexpression of AEBP1 on body weight and total body lipid content and found no significant difference between the transgenic and control mice when the mice were fed a regular diet. Therefore, we assessed the effect on mice fed a high-fat diet (HFD) from age 3 to 32 weeks. NT ( 8 males and 20 females) and TG (9 males and 24 females) mice were weighed every week for 7 months. Strikingly, we noticed premature sudden death of 9 female TG mice during the 7-month trial (the earliest at age 13 weeks, 2 at 14 weeks, 1 each at 15, 19, 20,21 and 27 weeks, and the last at 30 weeks). This high frequency $(\sim 38 \%)$ of premature sudden death was observed only in the female transgenic mice; no mouse from the other 3 groups died. It appears that HFD had a small contribution to the sudden death in the female TG population: a high frequency ( 21\%, 12 of 56$)$ of female TG mice fed a regular diet also suffered premature sudden death (4 at 20 weeks, 3 at 23 weeks, and 1 each at 18, 19, 24, 25, and 27 weeks). The reason for the sudden death in the female TG mice is unclear, but analysis of macrophages, where the transgene is also overexpressed (18), indicates a possible defect in cholesterol efflux with increased pro-inflammatory cytokine secretion. Eventually, the transgenic mice exhibited atherosclerotic lesions in the root of the aorta (submitted).

Female TG mice that survived to the end of the 7-month HFD study became massively obese (Figure 1B). The growth rates of NT and TG female mice were similar up to the age of 12 weeks, and then the rate of TG female growth significantly increased in comparison to the rate of NT females. The difference in mass between NT and TG females was significant at 21 weeks of age, and by the end of the study (at 32 weeks), there was a 1.6-fold increase in the average mass per mouse for TG versus their NT counterparts. Food consumption closely followed the same trend as the mass of mice. The food consumption by the female TG mice was significantly increased during the 20th and 21st weeks, when a burst of weight gain was observed (Figure 1C). At 32 weeks of age TG females were eating approximately 1.3-fold more food. Thus, energy intake (kcal/day/100 g body weight) was similar in TG and NT females (Figure 1D). However, the relative mass gained 
per food consumed was significantly greater in TG females from 21 to 25 weeks of age (Figure 1E). These results indicate that massive obesity in female TG mice was not induced by hyperphagia but rather by increased feed efficiency (increase in body weight per kilocalorie eaten). No significant difference in weight gain and food consumption was observed in males. The relative weight of total WAT to body weight in TG females was about 1.5-fold greater than that of NT females or NT or TG males at the end of the 7-month HFD feeding (Figure 1F). We repeated the analyses of growth and food consumption rates with a second set of female mice, in which there was also a trend toward increasing masses of TG versus NT, culminating in a 1.3-fold increase at 32 weeks when the mice were eating approximately 1.3-fold more food. No such effects were observed when the mice were fed a standard diet. These results raise the possibility that the AEBP1 gene and diet interact to influence fat mass in a sex-specific manner.

As anticipated from the massive obesity in the HFD-fed TG females, serum leptin levels were about 2.4-fold higher than in NT littermates, and this is probably a consequence, not a driver, of the change. However, this difference is not statistically significant owing to the very large variability among animals. No significant difference in the amount of leptin message was observed when it was analyzed with adipose tissues from 18-week-old virgin mice fed a standard diet. These results suggest that leptin expression is not regulated by AEBP1. Obesity is usually associated with elevated blood levels of glucose, insulin, free fatty acids, and leptin. It has been proposed that these features of obesity are due to the presence of enlarged adipocytes (26). The absence of significant increases in leptin levels suggests that obesity in the HFD-fed TG females is not associated with hypertrophy. Total serum cholesterol and triacylglyceride levels were not significantly different between TG and NT mice at the end of the 7-month HFD feeding. However, TG females had an apparent increase ( 2 -fold) in insulin levels over NT littermates at the end of the 7-month HFD feeding. Again, this difference is not statistically significant owing to the very large variability among animals. Glucose levels were measured every 3 weeks during the 7-month HFD study and showed no significant difference in the fed or fasted levels between TG and NT females. Results of intraperitoneal glucose and insulin tolerance tests for systemic insulin sensitivity indicate that systemic insulin sensitivity in the HFD-fed TG females was not compromised by the massive obesity. Again, these results suggest that the expanding fat mass may not be associated with adipocyte hypertrophy. It has been hypothesized that the size of adipocytes is inversely correlated with insulin sensitivity; namely, that larger adipocytes are associated with insulin resistance and smaller adipocytes are associated with insulin sensitivity $(27,28)$.

\section{AEBP1 Expression Is Modulated by Aging and Diet in a Sex-Specific Manner}

The sudden burst of weight gain in only the female TG mice at around 20 weeks of age suggests that AEBP1 may be regulated by sex-specific hormonal and metabolic changes associated with aging. We assessed whether the sudden rise in weight gain with increased feed efficiency in TG females was due to an increase in
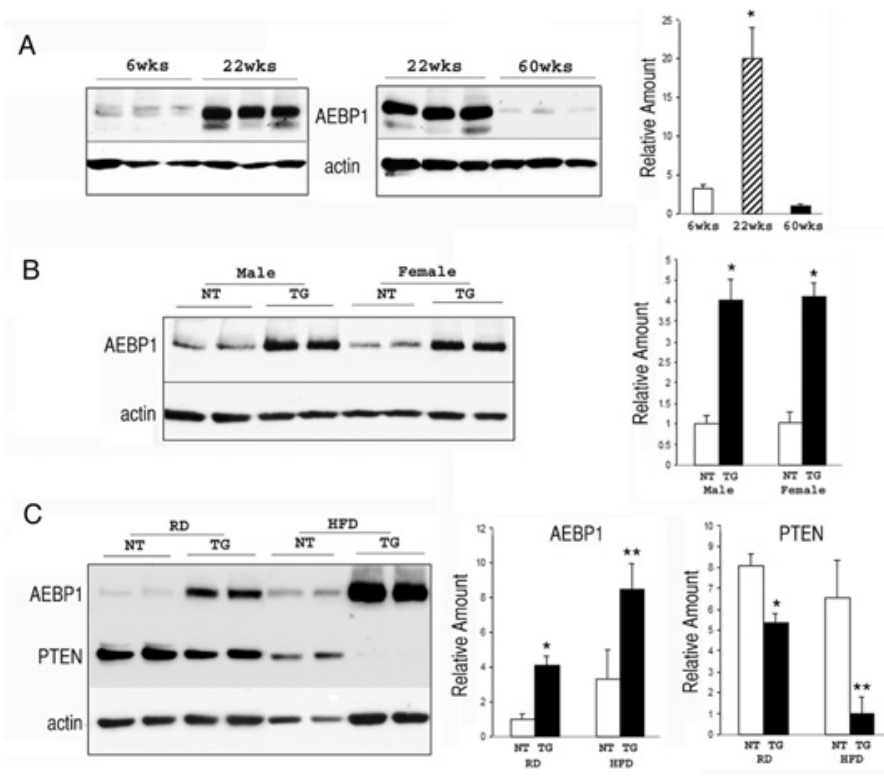

D
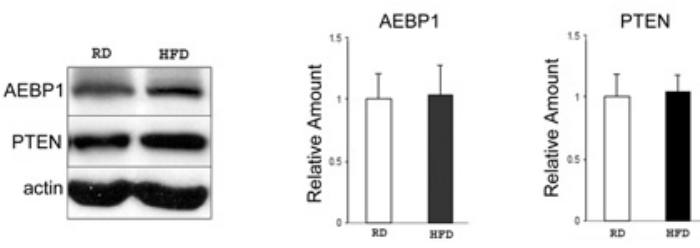

Figure 2. Age and diet modulate AEBP 1 expression in a sex-specific manner. (A) Expression of AEBP 1 was examined by immunoblotting with antiAEBPl antibody in female mice at 6 weeks $(n=4), 22$ weeks $(n=4)$, and 60 weeks ( $n=3$ ) of age. Actin is used as a loading control. The average values of AEBP1 expression in WAT at different ages are represented as mean \pm SD. ${ }^{*} P=0.0002$ (between 6 and 22 weeks); ${ }^{*} P=0.0005$ (between 22 and 60 weeks). (B) Expression of AEBP 1 was examined by immunoblotting with anti-AEBP1 antibody in regular diet-fed NT (male, $n=5$; female, $n=6$ ) and TG (male, $n=5$; female, $n=7$ ) mice at 32 weeks. Actin is used as a loading control. The average values of AEBP I expression in WAT from NT and TG mice are represented as mean \pm SD. ${ }^{*} P<0.0001$ compared with NT mice. (C) Expression of AEBPl and PTEN were examined by immunoblotting with anti-AEBPl or anti-PTEN antibody in NT (RD, $n=6$; HFD, $n=6$ ) and TG (RD, $n=7 ;$ HFD, $n=6$ ) females (32 weeks) that were fed on either regular diet (RD) or HFD for 29 weeks. Actin is used as a loading control. The average values of AEBPI and PTEN expression in WAT from NT and TG mice are represented as mean \pm SD. ${ }^{*} P<0.0001$ compared with RD-fed NT mice, ${ }^{* *} P \leq 0.0002$ compared with HFD-fed NT mice. (D) Expression of AEBPl and PTEN were examined by immunoblotting with anti-AEBP1 or anti-PTEN antibody in NT males (30 to 32 weeks) that were fed on either RD $(n=5)$ or HFD $(n=5)$ for 20 to 29 weeks. Actin was used as a loading control. The average values of AEBP1 and PTEN expression in WAT from NT mice are represented as mean \pm SD.

the endogenous AEBP1 levels with advancing age. Indeed, the amount of AEBP1 protein in NT mice was highly increased at 22 weeks of age over the level at 6 weeks, and then decreased to below the 6-week level when mice were approximately 1 year old (Figure 2A). These results suggest that the age-dependent expression of AEBP1 may be, in part, responsible for massive obesity in female TG mice. However, age-associated AEBP1 expression was also observed in male NT mice, in which the level at around 20 weeks of age was significantly increased from the 6-week level; unlike the results in female mice, the AEBP1 
expression did not decrease when the male mice were approximately 1 year old. These results suggest that the age-associated increase in AEBP1 expression is not sufficient for massive obesity in the female TG mice. Nevertheless, the rising incidence and severity of obesity seen in older individuals of many species are possibly mediated by a dynamic change in AEBP1 levels during aging, which can promote obesity associated with adipocyte hyperplasia. Next, we examined whether AEBP1 expression is further modulated by HFD in a sex-specific manner. The steady-state levels of AEBP1 proteins in the WAT of TG mice fed a standard diet were about 4-fold higher than the levels in NT mice for both sexes at the age of 32 weeks (Figure 2B). Although the AEBP1 levels at this age were similar in males and females, the amount of AEBP1 was markedly increased in female mice following HFD feeding for 7 months (Figure 2C). Indeed, the AEBP1 level was about 8.5-fold higher in the WAT of HFD-fed TG female mice than in standard diet-fed NT female mice. This increment is approximately similar to the sum of increments due to HFD induction of endogenous AEBP1 in NT mice ( $\sim 3.5$-fold) and the transgene expression ( $\sim 4$-fold). As anticipated, the amount of PTEN protein was inversely correlated with the amount of AEBP1: the amount of PTEN protein in the WAT of female TG mice fed a HFD was drastically reduced to about $10 \%$ of the amount in NT mice fed a standard diet (Figure 2C). In contrast, AEBP1 and PTEN levels remained unchanged in male mice after HFD feeding (Figure 2D). Together, our results suggest that the massive obesity observed only in the female TG mice was due to a combination of the ageand sex-specific HFD-mediated induction of endogenous AEBP1 expression, which resulted in a marked increase in the overall level of AEBP1 expression and a drastic reduction of PTEN protein in the WAT of female TG mice.

Recently, Kurlawalla-Martinez et al. (29) described phenotypes associated with adipose tissue-specific PTEN deletion, which was achieved by crossing Pten ${ }^{l x P / l x P}$ mice with adipose tissue-specific fatty acid binding protein $(a \mathrm{P} 2-\mathrm{Cre})$ transgenic mice. Kurlawalla-Martinez et al. demonstrated increased glucose tolerance and insulin sensitivity, with no effect on adiposity. The apparent discrepancy with our findings may be due to variability in tissue-specific targeting or differential regulation of the transgene by the aP2 promoter and upstream regulatory sequence, which also target to brown adipose tissue (BAT) and macrophages with variable levels of expression. Therefore, we cannot rule out the possibility that changes in PTEN levels in BAT and macrophages might also contribute to overall adiposity and insulin sensitivity. It has been suggested that obesity is associated with accumulation in adipose tissue of macrophages, which participate in inflammatory pathways that are activated in adipose tissues of obese individuals (30). Moreover, it has been demonstrated that adipocyte-specific inactivation of the insulin receptor gene in FIRKO mice produces selective insulin resistance in the adipose tissue, but does not affect whole-body glucose metabolism (31). It has been suggested that skeletal muscle accounts for $70 \%$ to $90 \%$ of glucose disposal following a carbohydrate load (32). Several knockout mouse models generated by tissue-specific disruption of insulin signaling in WAT, BAT, or skeletal muscle
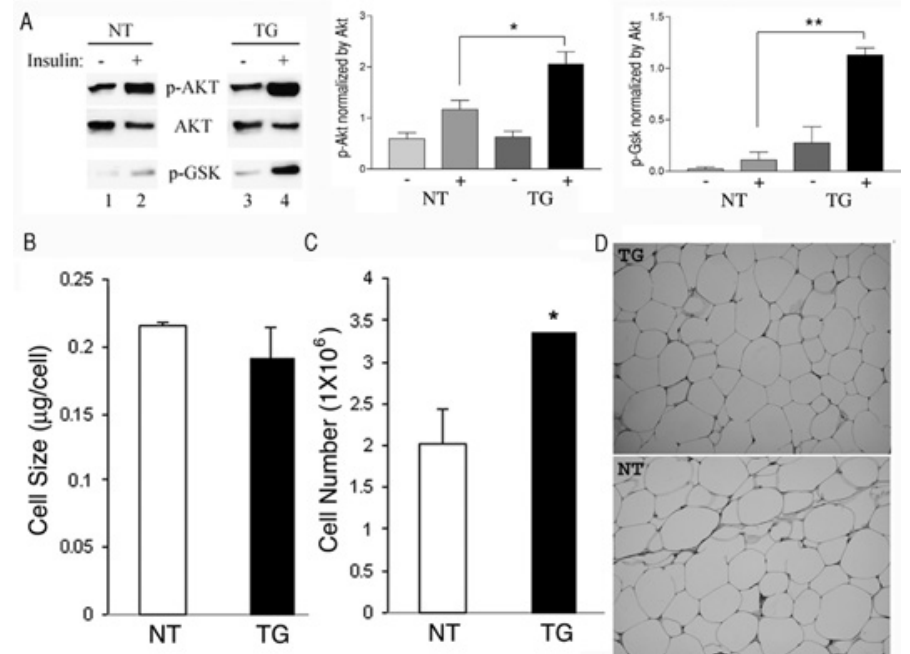

C
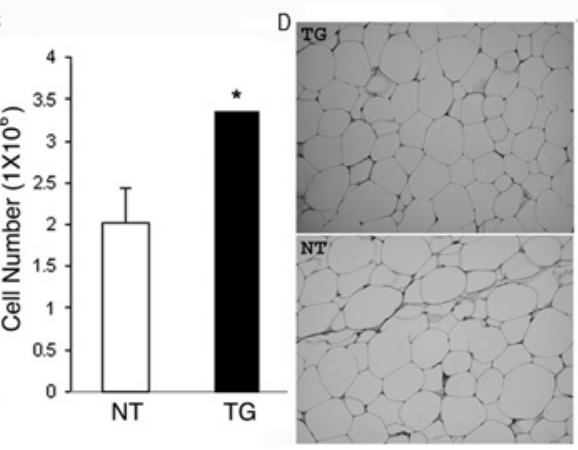

Figure 3. Increased survival signal in WAT of TG mice resulted in adipocyte hyperplasia. (A) The levels of phosphorylated Akt (p-AKT),

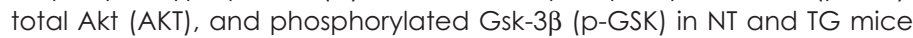
were measured before (-) and after (+) insulin injection by immunoblotting with respective antibody. The average values of $\mathrm{p}$-AKT (middle panel) and p-GSK (right panel) levels after normalized with AKT are represented as mean \pm SEM. ${ }^{*} P<0.05$ for NT $(n=5)$ and TG $(n=4),{ }^{* *} P=0.01$ for NT and TG $(n=3)$. (B) The fat cell size, which was expressed in milligrams of lipid per cell, was measured by counting the total number of osmium tetroxide-fixed cells obtained from a known weight of tissue lipid. NT, $n=2$; TG, $n=2$. (C) The total number of adipocytes in $0.775 \mathrm{~g}$ mean weight of WAT from TG $(n=2)$ mice and $0.515 \mathrm{~g}$ mean weight of WAT from NT $(n=2)$ mice are shown in millions of cells. ${ }^{*} P<0.05$. (D) WAT histology showing unilocular cells in paraffin sections stained with hematoxylin and eosin.

(33-36) showed significant differences in the whole-body glucose metabolism, in which insulin resistance induced even in a single tissue can contribute to major differences in phenotype. Finally, it has been suggested that strain-related distinction (FVB/N compared with C57BL/6J) in lipid handling is an important player in the differences in diabetic phenotype and insulin sensitivity (37).

\section{Hyperactivation of Akt With Adipocyte Hyperplasia in WAT of TG Mice}

Because PTEN protein is significantly reduced in the WAT of TG mice, we anticipated that the PI3K/Akt survival signal in TG mice would be correspondingly increased. To evaluate the mechanisms involved in AEBP1 action on PTEN and PI3K activation in TG mice, the inferior vena cava was injected directly with insulin to stimulate PI3K activation in vivo, and the status of Akt protein phosphorylation was determined by immunoblotting. Figure $3 \mathrm{~A}$ shows that TG animals contain higher amounts of phosphorylated Akt than the amounts in NT mice when stimulated by exogenous insulin. The increased phosphorylation of Akt in insulin-treated TG mice reflects hyperactivation of Akt as demonstrated by increased phosphorylation of its substrate GSK-3 $\beta$ in TG mice. These results suggest that AEBP1 can modulate in vivo adiposity by regulating PTEN functions that eventually influence the survival signaling pathway in WAT. 

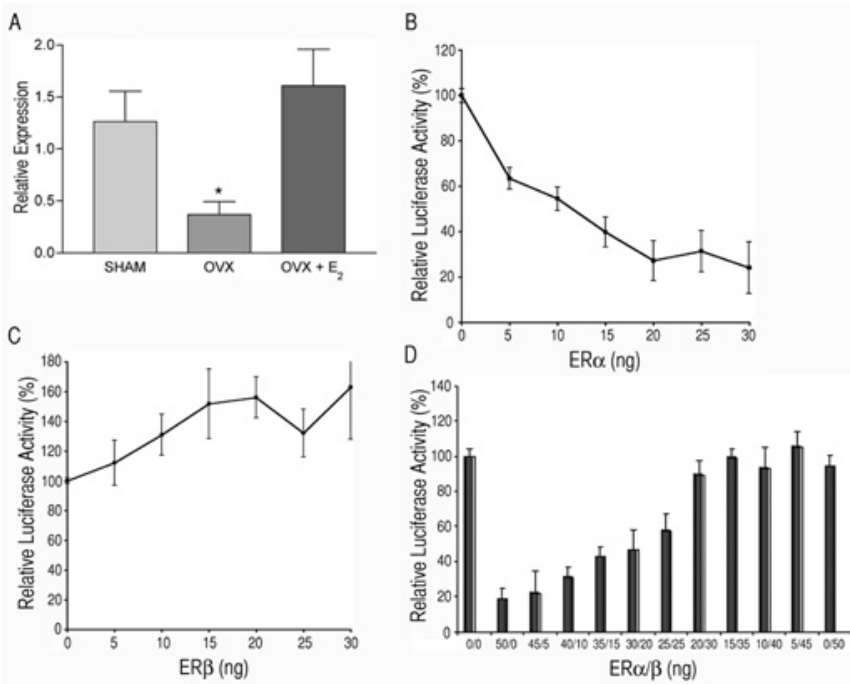

Figure 4. Estrogen/ER modulates AEBP1 expression in vivo and in vitro. (A) The average values of AEBPI expression in WAT from sham-operated (SHAM, $n=6$ ), ovariectomized (OVX, $n=5)$, or estradiol-injected OVX $\left(\mathrm{OVX}+\mathrm{E}_{2}, \mathrm{n}=6\right)$ mice are represented as mean $\pm \mathrm{SEM}$. ${ }^{*} P<0.05 \mathrm{com}-$ pared with sham-operated mice. (B) Dose-dependent repression of luciferase activity by ER $\alpha$. Each data point represents the mean \pm SEM luciferase activity measured in duplicate dishes for 4 independent experiments. The values are normalized with $\beta$-galactosidase activity. (C) Dose-dependent activation of the luciferase activity by ER $\beta$. Each data point represents the mean \pm SEM luciferase activity measured in duplicate dishes for 3 independent experiments. The values are normalized with $\beta$-galactosidase activity. (D) Inhibition of the ER $\alpha$ repression activity on the reporter expression by ER $\beta$. Each data point represents the mean \pm SEM luciferase activity measured in duplicate dishes for 2 independent experiments. The values are normalized with $\beta$-galactosidase activity.

Increased cell survival signal in the WAT of TG mice would result in increased fat cell number. To assess the contribution of adipocyte hyperplasia versus hypertrophy to increased adipose tissue mass in female TG mice, fat cell size and number were determined. The average cell size for TG mice $(0.191 \mu \mathrm{g} /$ cell $)$ was smaller than for NT littermates $(0.215 \mu \mathrm{g} /$ cell) (Figure 3B). Fat cell density, on the other hand, was increased in TG mice by 1.1-fold compare with NT littermates. Because the average weight of WAT in TG mice was 1.5-fold greater than that of NT littermates, the total cell number in TG mice was 1.65-fold greater than that of NT littermates (Figure 3C). Histological examination of the fat pads did not reveal any obvious enlargement of fat cells in TG mice (Figure 3D). These findings indicate that expansion of adipose tissue mass in the 32-week-old HFD-fed TG females was due to adipocyte hyperplasia and not to an increase in fat cell size. Therefore, the increased adiposity in the HFD-fed TG females may be mediated by a combined effect of increased proliferative potential of preadipocytes and promotion of survival signal on differentiating fat cells. However, the underlying molecular mechanisms by which aging and copious calorie consumption modulate AEBP1 levels in a sex-specific manner are not understood.

\section{Estrogen Modulates AEBP1 Expression}

Evidence from both humans and animals suggests that estrogen plays an important role in WAT regulation. Ovariectomy of rodents increases WAT, and estrogen replacement decreases WAT $(38,39)$. Similarly, postmenopausal women have increased WAT, and estrogen therapy decreases WAT levels compared with untreated postmenopausal women (40). The dynamic changes in AEBP1 levels with aging and the sex-specific diet effect on AEBP1 expression suggest that hormonal and metabolic changes associated with sexual maturation and aging affect AEBP1, and that AEBP1 may be a critical mediator of hormonal effects on adipose tissue homeostasis. To test this possibility we examined AEBP1 expression in ovariectomized (OVX) mice. Figure 4A shows that the steady-state level of AEBP1 was significantly reduced in OVX mice, and when mice were injected with $17 \beta$-estradiol $\left(\mathrm{E}_{2}\right)$, AEBP1 returned to the level in sham-operated mice. These results strongly suggest that AEBP1 expression is regulated by estrogen. However, our results are paradoxical concerning the role of estrogen in modulation of adiposity in OVX mice and postmenopausal women. The underlying mechanism in modulation of adiposity mediated by AEBP1 overexpression in TG mice may be distinct from the changes in OVX mice and postmenopausal women. Interestingly, changes in WAT induced by ovariectomy and/or estrogen treatment predominantly involved changes in adipocyte size in adults, when generation of new adipocytes is minimal $(38,41,42)$. In contrast, the increased WAT in AEBP1 ${ }^{\mathrm{TG}}$ mice is mainly mediated by adipocyte hyperplasia. These findings suggest that the decreased AEBP1 expression in OVX mice may have a distinct role in adipocyte hypertrophy.

In general, estrogens are assumed to mediate their effects through 2 specific nuclear receptor isoforms called estrogen receptor (ER) $\alpha$ and ER $\beta$. Increases in WAT were also seen in ER $\alpha$ knockout (ER $\alpha-\mathrm{KO})$ mice with advancing age in both sexes (43). Energy intake was similar in wild-type and ER $\alpha-\mathrm{KO}$ male mice, indicating that obesity was not the result of hyperphagia. These findings suggest that increases in WAT after estrogen deficiency and decreases in WAT after estrogen replacement predominantly reflect changes in signaling through $\mathrm{ER} \alpha$, although $\mathrm{ER} \beta$ may play a role. The loss of estrogen/ER $\beta$ signaling resulted in phenotypical and biochemical changes in adipose tissues opposite from those of estrogen/ER $\alpha$ signaling (44). The ER subtypes have different tissue distribution (45) and exert, as homodimers or heterodimers, transcriptional regulation of different target genes through estrogen responsive elements (ERE; 46). Sequence analysis of the proximal promoter region $(\sim 2 \mathrm{~kb})$ of the AEBP1 gene identified several copies of the half-palindromic ERE that may be involved in the regulation of AEBP1 expression. We tested whether ER directly regulates AEBP1 expression by cotransfection experiments. When the reporter construct $p(-1845 /+206)$ AEBP1-LUC containing the 2-kb DNA fragment driving the reporter gene luciferase was transfected along with an expression vector encoding $E R \alpha$, the promoter activity was markedly repressed in a dose-dependent manner (Figure 4B). These results are consistent with the increased WAT in both ER $\alpha-\mathrm{KO}$ and AEBP1 ${ }^{\mathrm{TG}}$ mice. The increased WAT in ER $\alpha-\mathrm{KO}$ mice involves both adipocyte hyperplasia and hypertrophy that may result from changes in the differentiation and/or proliferation of the mesenchymal/preadipocyte/adipocyte lineage (43). Our results suggest that AEBP1 mediates the ER $\alpha$ signaling on the hyperplastic 
modulation of adiposity. Indeed, it has been shown previously that $\mathrm{E}_{2}$ increases human preadipocyte proliferation in vitro $(47,48)$, and overexpression of AEBP1 in 3T3-L1 preadipocytes increases their proliferation rate (10). Together, our results suggest that AEBP1 has 2 distinct estrogen-mediated regulatory roles in modulation of adiposity via fat cell number and fat cell size.

The decreased AEBP1 level in OVX mice and subsequent return to the level in sham-operated mice with $\mathrm{E}_{2}$ injection suggest that the ligand-stimulated ER should function as a trans-activator of AEBP1 expression, in which ER $\beta$ may be responsible for the positive role to counteract the repression activity of ER $\alpha$. ER $\beta$ has been reported to inhibit the transcriptional activity of $\mathrm{ER} \alpha$, possibly through ER $\alpha$ /ER $\beta$ heterodimer formation (49), thus suggesting that the biological response to estrogens is dependent on the relative ratios of ER $\alpha$ and ER $\beta$ levels in cells. Indeed, a significant induction of the reporter activity was observed when the reporter plasmid $\mathrm{p}(-1845 /+206)$ AEBP1-LUC was cotransfected with a plasmid expressing ER $\beta$ (Figure $4 \mathrm{C}$ ). Moreover, the repression activity of $E R \alpha$ was inhibited in a dose-dependent manner as the ER $\alpha / E R \beta$ ratio in the transfected cells decreased (Figure 4D). These results suggest that the overall net effect of ligand-stimulated ER in WAT is dependent on the relative amounts of the 2 specific isoforms of ER. Together, our results suggest that AEBP1 may be a critical mediator of the hormonal effects on adipose tissue homeostasis through signaling of both ER $\alpha$ and ER $\beta$.

In this article, we have demonstrated that AEBP1 has a key role in modulation of in vivo adiposity. Detailed analysis of the transgenic mice indicates that AEBP1 may be a novel regulator of adiposity and energy balance in mammals. The present study clearly indicates that persistent overexpression of AEBP1 during adipocyte differentiation promotes HFD-induced obesity with increased in feed efficiency in female mice. Our data underscore a metabolic facet of AEBP1, which may operate as a novel effector of estrogen function linking energy metabolism. It has been well established that sex steroid hormones are involved in the regulation of metabolism, distribution, and accretion of adipose tissues. Homeostasis of adipose tissue requires proper amounts of estrogens and androgens. In an aging population, as the levels of sex steroid hormones decrease, frequency of central obesity increases. Apparent positive effects on central obesity have been observed in older women by hormone replacement therapy and in aging men by testosterone therapy, with numerous side effects including bone loss and an increased risk for coronary heart disease, stroke, thromboembolic events, breast cancer, and prostate cancer (50). Our results suggest that AEBP1 may be a novel effector of estrogen action that is specific for adipose tissues and pathways related to lipid metabolism on the regulation of central obesity. Understanding the role AEBP1 plays in this regulation will lead to the eventual identification of potential molecular targets that may lead to novel methods of anti-obesity therapies.

\section{ACKNOWLEDGMENTS}

We are indebted to Bradford Lowell and Joel A. Lawitts for their support in this work, which was initiated during H.-S.R.'s sabbatical leave from the Department of Medicine, Division of Endocrinology, Beth
Israel Deaconess Medical Center and Harvard Medical School. This work was supported by grants from the Canadian Diabetes Association, the Heart and Stroke Foundation (Nova Scotia) of Canada (HSFC), the Natural Sciences and Engineering Research Council (NSERC) of Canada, and Canadian Institutes of Health Research (CIHR) to H.S.R. We acknowledge the support of the HSFC Visiting Scientist Award to H.S.R. and the Postdoctoral Fellowship Program of Korea Science \& Engineering Foundation (KOSEF) to H.-J.L.

Address correspondence and reprint requests to Hyo-Sung Ro, Department of Biochemistry \& Molecular Biology, Faculty of Medicine, Dalhousie University, Tupper Medical Building, 1850 College Street, Halifax, NS, B3H IX5 Canada. Phone: 902-494-2367; fax 902-494-1355; e-mail: hsro@dal.ca.

Submitted September 18, 2005; accepted for publication October 24, 2005.

\section{REFERENCES}

1. Flier JS. (2004) Obesity wars: molecular progress confronts an expanding epidemic. Cell 116:337-50.

2. Reitman ML. (2004) Magic bullets melt fat. Nat. Med. 10:581-2.

3. Kolonin MG, Saha PK, Chan L, Pasqualini R, Arap W. (2004) Reversal of obesity by targeted ablation of adipose tissue. Nat. Med. 10:625-32.

4. Distel RJ, Ro H-S, Rosen BS, Groves DL, Spiegelman BM. (1987) Nucleoprotein complexes that regulate gene expression in adipocyte differentiation: direct participation of c-fos. Cell 49:835-44.

5. Herrera R, Ro H-S, Robinson GS, Xanthopoulos KG, Spiegelman BM. (1989) A direct role for C/EBP and the AP-1-binding site in gene expression linked to adipocyte differentiation. Mol. Cell. Biol. 9:5331-9.

6. Ro H.-S., Roncari D.A.K. (1991) The C/EBP-binding region and adjacent sites regulate expression of the adipose P2 gene in human preadipocytes. Mol. Cell. Biol. 11:2303-6.

7. He G-P, Muise A, Wu-Li A, Ro H-S. (1995) A eukaryotic transcriptional repressor with carboxypeptidase activity. Nature 378:92-6.

8. Muise AM, Ro H-S. (1999) Enzymic characterization of a novel member of the regulatory B-like carboxypeptidase with transcriptional repression function: stimulation of enzymic activity by its target DNA. Biochem. J. 343:341-5.

9. Park J-G, Muise A, He G-P, Kim S-W, Ro H-S. (1999) Transcriptional regulation by the gamma5 subunit of a heterotrimeric $G$ protein during adipogenesis. EMBO J. 18:4004-12.

10. Kim S, Muise A, Lyons PJ, Ro H-S. (2001) Regulation of adipogenesis by a transcriptional repressor that modulates MAPK activation. J. Biol. Chem. 276:10199206.

11. Lyons PJ, Muise AM, Ro H-S. (2005) MAPK modulates the DNA binding of adipocyte enhancer-binding protein 1. Biochemistry 44:926-31.

12. Ro H-S, Kim S-W, Wu D, Webber C, Nicholson TE. (2001) Gene structure and expression of the mouse adipocyte enhancer-binding protein. Gene 280: 123-33.

13. Layne MD et al. (1998) Aortic carboxypeptidase-like protein, a novel protein with discoidin and carboxypeptidase-like domains, is up-regulated during vascular smooth muscle cell differentiation. J. Biol. Chem. 273:15654-60.

14. Gorbenko $O$ et al. (2004) Identification of a novel binding partners for tumor suppressor PTEN by a yeast two-hybrid approach. Exp. Oncol. 26:15-9.

15. Sulis ML, Parsons R. (2003) PTEN: from pathology to biology. Trends Cell Biol. 13:478-83.

16. Goberdhan DCl, Wilson C. (2003) PTEN: tumor suppressor, multifunctional growth regulator and more. Human Mol. Genet. 12:R239-48.

17. Ross SR et al. (1990) A fat-specific enhancer is the primary determinant of gene expression for adipocyte P2 in vivo. Proc. Natl. Acad. Sci. U. S. A. 87:9590-4

18. Makowski L et al. (2001) Lack of macrophage fatty-acid-binding protein aP2 protects mice deficient in apolipoprotein E against atherosclerosis. Nat. Med. 7:699-705.

19. Kuksis A, Myher JJ, Marai L, Geher K. (1976) Estimation of plasma free fatty acids as the trimethylsilyl (TMS) esters. Anal. Biochem. 70:302-12.

20. Hirsch J, Gallian E. (1968) Methods for the determination of adipose cell size in man and animals. J. Lipid Res. 9:110-9.

21. He G-P, Kim S, Ro H-S. (1999) Cloning and characterization of a novel zinc finger transcriptional repressor: a direct role of the zinc finger motif in repression. J. Biol. Chem. 274:14678-84.

22. Prins JB et al. (1997) Tumor necrosis factor- $\alpha$ induces apoptosis of human adipose cells. Diabetes 46:1939-44.

23. Gagnon A, Dods P, Roustan-Delatour N, Chen C-S, Sorisky A. (2001) 
Phosphatidylinositol-3,4,5-trisphosphate is required for insulin-like growth factor 1-mediated survival of 3T3-L1 preadipocytes. Endocrinology 142:205-12.

24. Longo KA, Kennell JA, Ochocinska MJ, Ross SE, Wright WS, MacDougald OA. (2002) Wnt signaling protects 3T3-L1 preadipocytes from apoptosis through induction of insulin-like growth factors. J. Biol. Chem. 277:38239-44.

25. Fischer-Posovszky P, Tornqvist H, Debatin KM, Wabitsch M. (2004) Inhibition of death-receptor mediated apoptosis in human adipocytes by the insulin-like growth factor I (IGF-I)/IGF-I receptor autocrine circuit. Endocrinology 145:1849-59.

26. Van Harmelen $\vee$ et al. (1998) Leptin secretion from subcutaneous and visceral adipose tissue in women. Diabetes 47:913-7.

27. Okuno A et al. (1998) Troglitazone increases the number of small adipocytes without the change of white adipose tissue mass in obese Zucker rats. J. Clin. Invest. 101:1354-61.

28. Kubota $\mathrm{N}$ et al. (1999) PPAR gamma mediates high-fat diet-induced adipocyte hypertrophy and insulin resistance. Mol. Cell 4:597-609.

29. Kurlawalla-Martinez C, Stiles B, Wang Y, Devaskar SU, Kahn BB, WU H. (2005) Insulin hypersensitivity and resistance to streptozotocin-induced diabetes in mice lacking PTEN in adipose tissue. Mol. Cell. Biol. 25:2498-510.

30. Weisberg SP, McCann D, Desai M, Rosenbaum M, Leibel RL, Ferrante AW Jr. (2003) Obesity is associated with macrophage accumulation in adipose tissue. J. Clin. Invest. 112:1796-808.

31. Bluher M, Michael MD, Peroni OD, Ueki K, Carter N, Kahn BB, Kahn CR. (2002) Adipose tissue selective insulin receptor knockout protects against obesity and obesity-related glucose intolerance. Dev. Cell 3:25-38.

32. DeFronzo RA. (1997) Pathogenesis of type 2 diabetes: metabolic and molecular implications for identifying diabetes genes. Diabetes Rev. 5:177-269.

33. Abel ED et al. (2001) Adipose-selective targeting of the GLUT4 gene impairs insulin action in muscle and liver. Nature 409:729-33.

34. Bruning JC et al. (1998) A muscle-specific insulin receptor knockout exhibits features of the metabolic sysdrome of NIDDM without altering glucose tolerance. Mol. Cell 2:559-69.

35. Zisman A et al. (2000) Targeted disruption of the glucose transporter 4 selectively in muscle causes insulin resistance and glucose intolerance. Nat. Med. 6:924-8.

36. Guerra C et al. (2001) Brown adipose tissue-specific insulin receptor knockout shows phenotype without insulin resistance. J. Clin. Invest. 108: 1205-13.
37. Haluzik $M$ et al. (2004) Genetic background (C57BL/6J versus FVB/N) strongly influences the severity of diabetes and insulin resistance in ob/ob mice. Endocrinology 145:3258-64

38. Wade GN, Gray JM, Bartness, TJ. (1985) Gonadal influence on adiposity. Int. J. Obes. 9:83-92

39. Pederson SB, Borglum JD, Eriksen EF, Richelsen B. (1991) Nuclear estradiol binding in rat adipocytes: regional variations and regulatory influences of hormones. Biochim. Biophys. Acta 1093:80-6.

40. Tchernof A, Calles-Escandon J, Sites CK, Poehlman ET. (1998) Menopause, central body fatness, and insulin resistance: effects of hormone-replacement therapy. Coron. Artery Dis. 9:503-11

41. Pederson SB, Borglum JD, Moller-Pederson T, Richelsen B. (1992) Effects of in vivo estrogen treatment on adipose tissue metabolism and nuclear estrogen receptor binding in isolated rat adipocytes. Mol. Cell. Endocrinol. 85:13-9.

42. Crandall DL, Busler DE, Novak TJ, Weber RV, Kral JG. (1998) Identification of estrogen receptor $\beta$ RNA in human breast and abdominal subcutaneous adipose tissue. Biochem. Biophys. Res. Comm. 248:523-6.

43. Heine PA, Taylor JA, Iwamoti GA, Lubahn DB, Cooke PS. (2000) Increased adipose tissue in male and female estrogen receptor-alpha knockout mice. Proc. Natl. Acad. Sci. U. S. A. 97:12729-34.

44. Naaz A et al. (2002) Effect of ovariectomy on adipose tissue of mice in the absence of estrogen receptor alpha (ERalpha): a potential role for estrogen receptor beta (ERbeta). Horm. Metab. Res. 34:758-63.

45. Kuiper GG et al. (1997) Comparison of the ligand binding specificity and transcript tissue distribution of the estrogen receptors $\alpha$ and $\beta$. Endocrinology 138:863-70.

46. Cowley SM, Hoare S, Mosselman S, Parker MG. (1997) Estrogen receptors alpha and beta form heterodimers on DNA. J. Biol. Chem. 272:19858-62.

47. Anderson LA, McTernan PG, Barnett AH, Kumar S. (2001) The effects of androgens and estrogens on pradipocyte proliferation in human adipose tissue: influence of gender and site. J. Clin. Endocrinol. Metab. 86:5045-51.

48. Roncari DAK, Van RL. (1978) Promotion of human adipocyte precursor replication by $17 \beta$-estradiol in culture. J. Clin. Invest. 62:503-8.

49. Hall JM, MCDonnell DP. (1999) The estrogen receptor $\beta$ isoform (ER $\beta$ ) of the human estrogen receptor modulates ER $\alpha$ transcriptional activity, and is a key regulator of the cellular response to estrogens and antiestrogens. Endocrinology 140:5566-78.

50. Nelson HD, Humphrey LL, Nygren P, Teutsch SM, Allan JD. (2002) Postmenopausa hormone replacement therapy: scientific review. JAMA 288:872-81. 